\section{FP9 (continued)}

Description: After identifying a skill deficit in handling and preparing fresh produce in school kitchens, a training was developed by a culinary chef and taught to 562 employees throughout the state. Additionally, all Idaho school kitchens received a set of laminated vegetable scratch cooking fact sheets titled "Fresh ideas for Fresh Vegetables". These fact sheets identified valuable information such as yields, storage, preparation, and recommended seasonings for a variety of vegetables. The front of each fact sheet included an image and educational facts and could be used as a visual marketing tool when that vegetable was featured in a meal.

Evaluation: Participants were given an evaluation at the conclusion of the workshop that required their response to a variety of questions involving their reaction to the workshop and covered material. The evaluation also had them rate their proficiency both before and after the workshop, as well as indicate the timeframe in which they planned to use the newly gained knowledge and skills.

Conclusions and Implications: The first step in increasing the amount of fresh produce served in school meals is developing confidence, skills, and resources necessary to safely and efficiently prepare a variety of fresh produce. Confidence level was evaluated by having participants rate their skill level using a scale of 1-5, from Beginner (1) to Expert (5), both before and after the workshop. Before training, only $16 \%$ of participants rated their skill at a level 4 or higher, however after the training, 79\% rated their skill at that same level. Additionally, 95\% of participants indicated they would begin to use their new knowledge immediately.

Funding: USDA Grant \#CNTN-12-ID.

\section{FP10 Withdrawn}

\section{FP11 Innovative Strategies for Creating Smarter Lunchrooms in Montana High Schools} Katie Bark, RD, LN, SNS, kbark@mt.gov, Montana Team Nutrition, Montana State University, 202 Romney Hall, Bozeman, MT 59715; C. Byker-Shanks, PhD, Montana State University; M. Stenberg, $R D, L N$, Montana Team Nutrition

Objective: Participants will understand Smarter Lunchroom strategies successfully piloted in high school cafeterias and be able to measure success of changes through plate waste data collection, student selection and meal participation rates.

Description: Smarter Lunchrooms integrates behavioral economics with food marketing techniques to trigger students to consume healthier foods. Strategies implemented in the school cafeteria "nudge" students to choose healthier items. This poster describes an innovative approach for incorporating Smarter Lunchroom strategies at the high school level to encourage increased consumption and less waste of fruits and vegetables. It is based on a pilot project conducted with five high schools in Montana during the 2014-2015 school year. Project results indicate that the success of the implementation of Smarter Lunchroom strategies can be enhanced through a team approach allowing for staff and students to share perspectives and create actionable solutions. Smarter Lunchroom best practices and lessons learned from the project will be shared.

Evaluation: The pilot project was evaluated by measuring several factors in the school nutrition program at each of the five high schools including a plate waste data collection, student selection and meal participation rates. Observational data was also collected from the members of the Smarter Lunchroom Advisory Teams at each school.

Conclusions and Implications: The results from this pilot project with five Montana high schools indicate the value of implementing Smarter Lunchroom strategies to improve student participation and decrease food waste. An implication learned from this project demonstrates a need to help schools sustain a continual dialogue between students and staff regarding school lunch improvements. Funding: USDA Grant \#CNTN-MT-14, Cornell University, Behavioral Economics Center.

\section{FP12 Maryland's Building Blocks for Healthy Children: Increasing the Variety and Acceptance of Fruits, Vegetables, and Whole- Grains}

MeganLopes, MPH, megan.sweatlopes@maryland.gov, Maryland State Department of Education, 200 West Baltimore Street, Baltimore, MD 21201; B. Schuler, MSW, University of Maryland Baltimore; M. Black, PhD;

K. Brown, MA, Maryland Family Network; J. Dzimiera, RD, $L D N, M E d$, Maryland State Department of Education

Objective: Describe the nutrition and physical activity environment in CACFP child care centers. State fruit and vegetable preferences of 3-5 year olds. Describe staff demographics, and beliefs and practices about nutrition and physical activity. Describe eating habits of 3- to 5-yearolds and quality of lunches served (via meal observations). Description: The Institute of Medicine (IOM) and 2010 Dietary Guidelines for Americans have outlined recommendations to improve the variety and amount of fruits, vegetables, and whole-grains, and to improve the environment of child care centers. Aligned with these recommendations, Maryland's 2013 Team Nutrition grant recruited 22 CACFP child care centers and provided a six month course focusing on enhancing the nutrition and physical activity environment for child care directors and or the CACFP program coordinators. Also, Nutrition Navigators provided nutrition education to children and technical assistance to staff in child care centers during this time.

Evaluation: Paper surveys were distributed to child care center staff and parent/caregivers for 3-5 year old children. These surveys gathered demographic information and nutrition and physical activity beliefs and practices. A child care environment assessment was completed utilizing the Environment Policy and Assessment Observation (EPAO) tool to objectively and effectively describe the 